\title{
Mikroskopische Kolitis
}

\section{Patienten haben hohen Leidensdruck}

— Bei anhaltenden wässrigen unblutigen Diarrhöen sollte immer an eine mikroskopischen Kolitis gedacht werden. „Da der endoskopische Befund bei den betroffenen Patienten unauffällig ist, sollten bei der Koloskopie immer Stufenbiopsien entnommen werden", sagte Prof. Stephan Miehlke, Hamburg. Nur dadurch könne die Diagnose gesichert werden.

Typischerweise findet sich eine lymphoplasmazelluläre Inflammation der Lamina propria. Bei Nachweis eines verdickten subepithelialen Kollagenbandes spricht man von der kollagenen Kolitis, bei Vermehrung der intraepithelialen Lymphozyten von einer lymphozytären Kolitis. „Der Nachweis dieser Erkrankung ist wichtig, da die Patienten unter einem erheblichen Leidensdruck und einer deutlich reduzierten Lebensqualität leiden", so Miehlke.

Nach neueren Untersuchungen ist die Häufigkeit der mikroskopischen Kolitis durchaus vergleichbar mit der des Morbus Crohn und der Colitis ulcerosa. Die Ätiologie und Pathogenese sind ungeklärt. Diskutiert wird eine abnorme immunologische Reak-

tion auf exogene bzw. luminale Faktoren wie Infektionen, Gallensäuren und Medikamente bei genetisch prädisponierten Personen.

Die Therapie der Wahl bei der kollagenen Kolitis ist das topische Steroid Budesonid (Budenofalk ${ }^{\circledR}$ ). „Dabei handelt es sich um die beste evidenzbasierte Therapie für dieses Krankheitsbild", so Miehlke. Es führe zu einer raschen Symptombefreiung und

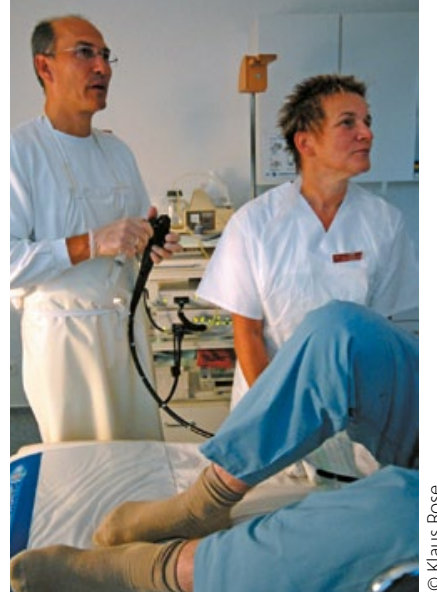

Koloskopie mit Stufenbiopsie auch bei unauffälligem Befund. einer Normalisierung

der Lebensqualität. Die Therapie sollte mit $9 \mathrm{mg}$ täglich über sechs bis acht Wochen begonnen werden. In entsprechenden randomisierten placebokontrollierten Studien konnte damit eine Erfolgsrate von fast $90 \%$ erreicht werden.

Doch in über $60 \%$ der Fälle kommt es innerhalb der ersten Wochen nach Thera- pieende zum Rezidiv. Dann sollte erneut eine Behandlung mit Budesonid (9 mg täglich) eingeleitet werden, bis eine Remission erzielt ist. Anschließend empfiehlt es sich, zur dauerhaften Remissionserhaltung die Budesonid-Therapie in möglichst niedrigen Dosierung (3-6 mg täglich) fortzuführen. Bei leichter Symptomatik oder additiv bei Bedarf können auch Antidiarrhoika eingesetzt werden. Der Stellenwert anderer Ansätze wie Aminosalicylate, Wismutsalze, Colestyramin, Immunsuppressiva und Anti-TNFalpha-Antikörper ist nicht gesichert.

- Dr. med. Peter Stiefelhagen

Quelle: Workshop und Pressegespräch „Microscopic Colitis - Creating Awareness for an Underestimated Disease", Basel, Mai 2012 (Veranstalter: Falk Foundation e.V.)

\section{Kombination aus Opioid und Opioidrezeptorantagonist \\ Stabile Analgesie bei starken chronischen Schmerzen}

- Starke Schmerzen benötigen eine effektive analgetische Therapie. Eine Kombination von einem stark wirksamen, retardierten Opioid mit einem Opioidrezeptorantagonisten kann stabile Plasmaspiegel bei gastrointestinaler Verträglichkeit ermöglichen.

In der Behandlung starker, chronischer Schmerzen haben moderne, lang wirksame Opioidanalgetika das pharmakologisch ungünstige Morphin weitgehend abgelöst. Entscheidender Faktor für die Auswahl eines Opioids ist neben der individuellen Symptomatik das Nebenwirkungsprofil, berichtete Prof. Dr. Gerd Mikus, Heidelberg. Die Unterschiede zwischen den Opioiden seien dadurch bedingt, dass jede Substanz eine charakteristische Affinität für die Opi- oidrezeptoren $(\mu, \kappa, \delta$ und ORL 1$)$ und eine entsprechende eigene intrinsische Aktivität an jedem dieser Rezeptoren habe. Die Fixkombination aus retardiertem Oxycodon und retardiertem Naloxon (Targin ${ }^{\circledR}$ ) biete den Vorteil einer hohen analgetischen Potenz bei zugleich guter gastrointestinaler Verträglichkeit, betonte Mikus.

\section{Problem Magenverweilzeit}

Eine wichtige Intention der Opioidtherapie ist das Erreichen möglichst konstanter und reproduzierbarer Plasmapiegel. Aufgrund der opioidinduzierten Verzögerung der Magenentleerung kann es aber zu Verzögerungen in der Resorption und damit zu Schwankungen der Wirkspiegel kommen, so Prof. Dr. Henning Blume, Oberursel.
Auch wenn die Tabletten nach der Mahlzeit eingenommen werden, sei die Magenentleerung und damit die Resorption des Opioids verlangsamt. Durch die Kombination von Oxycodon mit dem peripher wirksamen Opioidantagonisten Naloxon könne man das Problem an der Wurzel anpacken. Da der Antagonist die Wirkung des Opioids im Gastointestinaltrakt verhindert, sind laut Blume gleichmäßige Plasmaprofile mit der Folge einer verbesserten Wirksamkeit und Verträglichkeit zu erwarten.

\footnotetext{
- Abdol A. Ameri

Quelle: Symposium „Leitliniengerechte Schmerztherapie vs. Verordnungsdruck" im Rahmen des Deutschen Schmerz- und Palliativtages, Frankfurt, März 2012 (Veranstalter: Mundipharma)
} 